\title{
Mouvement de colère chez les sages-femmes
}

\section{Burst of anger in midwife}

\author{
E. Bailleux \\ C Springer-Verlag France 2011
}

Des sages-femmes, des étudiants sages-femmes et des usagers venus de toute la France ont manifesté ce 12 mai dernier à Paris. Soutenu par le Conseil national de l'ordre des sages-femmes, ce mouvement regroupait l'ensemble des organisations représentatives des sages-femmes.

Parmi les revendications exprimées, elles dénoncent les conditions de la naissance en France et défendent une réorganisation de la périnatalité replaçant la sage-femme au cœur du dispositif. Notre organisation actuelle des soins périnataux, contrairement à celle d'autres pays européens, montre en effet ses limites avec un bilan de morbidité-mortalité qui stagne depuis plusieurs années (au huitième rang européen). Le dernier Plan périnatalité mettait en avant l'importance de la place de la sage-femme dans le suivi des grossesses à faible risque, avec un accompagnement personnalisé et une continuité de la prise en charge. Depuis, quelques expériences satisfaisantes ont vu le jour dans nos maternités. Celles-ci restent néanmoins trop peu nombreuses. Les sages-femmes, rejoignant la demande des associations d'usagers, souhaitent la mise en place de filières physiologiques dont elles assureront la gestion, dans toutes les structures de naissance. En réponse, le ministère de la Santé envisagerait la mise en place d'un parcours incitatif pour les femmes enceintes vers les sages-femmes comme professionnels de premier recours.

Elles prônent également l'amélioration de la qualité de l'accompagnement des grossesses, des accouchements et des suites de naissance par l'augmentation du nombre de sages-femmes. Le défilé a d'ailleurs été ponctué d'un slogan fort : « une femme, une sage-femme ».

Le développement de structures alternatives sécurisées, répondant aux besoins exprimés par les usagers pour l'accompagnement de la grossesse et de l'accouchement, reste aussi à l'heure actuelle encore insuffisant.

Il existe un déséquilibre important dans l'exercice de la profession allant d'une pratique de type paramédical à une

\section{E. Bailleux $(\square)$}

École de sages-femmes du CHRU de Lille,

CHRU, F-59037 Lille cedex, France

e-mail : Elisabeth.bailleux@chru-lille.fr pratique médicale autonome, ce que certaines professionnelles ne peuvent exercer complètement. Cela est entretenu par la méconnaissance des compétences, des responsabilités et $\mathrm{du}$ cursus de formation des sages-femmes par les professionnels de santé avec qui elles travaillent. Rappelons que la profession de sage-femme est une profession médicale dont l'autonomie professionnelle est définie par la loi. Elles sont compétentes pour dépister la pathologie et ainsi faire appel aux obstétriciens quand la situation le nécessite. Leur champ de compétences s'est beaucoup élargi ces dernières années pour répondre aux besoins de santé actuels.

Les sages-femmes souhaitent garantir une réelle autonomie et la spécificité de la profession dans son mode d'exercice.

De même, la formation passe par un recrutement par le PCEM 1 depuis 2002 et entre dans le cadre de l'harmonisation européenne de l'organisation des études de santé par la mise en place du schéma Licence-Master-Doctorat. La première étape de l'application du processus de Bologne aux études de médecine, de maïeutique, d'odontologie et de pharmacie a été pour cette dernière année universitaire la mise en place de la première année commune aux études de santé (Paces). Celle des deuxième et troisième années de licence sera l'étape suivante. Des diplômes de formation générale (DFG) en sciences médicales, odontologiques et pharmaceutiques ont été créés avec des textes officiels publiés en avril. À ce jour, il est à déplorer le retard à la parution de l'arrêté régissant le nouveau programme « licence maïeutique ». Un engagement avait été pris au décours de la manifestation pour une sortie de ce texte avant l'été afin de permettre l'organisation de l'année scolaire prochaine dans les écoles...

Ce projet d'arrêté de DFG en sciences maïeutiques a reçu un avis favorable à l'unanimité de la part de la CCEN (Commission consultative d'évaluation des normes) le 7 juillet et, avec un peu de chance, il sera publié avant la rentrée de septembre!

Par ailleurs, la loi portant réforme de l'hôpital et relative aux patients, à la santé et aux territoires stipule que « $L a$ formation initiale des sages-femmes peut être organisée au sein des universités... ». Elle n'en a cependant pas fixé les modalités. 
L'Association des sages-femmes enseignantes françaises (ASFEF) ainsi que les représentants de l'Association nationale des étudiants sages-femmes (ANESF) réclament que cette intégration soit obligatoire. Afin d'en garantir la qualité et de préserver l'autonomie de la formation, ils demandent que soient créées des composantes universitaires à part entière selon le modèle d'école ou d'institut universitaire. Citons, pour exemple, la création de l'école universitaire de maïeutique de Marseille-Méditerranée en juillet 2010.

Enfin, la reconnaissance du niveau d'études à bac +5 impose une modification des statuts. Au regard des respon- sabilités et de la compétence médicale de la profession, celle-ci passe de fait par la revalorisation de la cotation des actes médicaux et en parallèle des salaires, quel que soit le mode d'exercice. Soulignons que, parmi les professions libérales de santé, la profession de sage-femme est la seule à avoir un revenu annuel en déclin! Tant en ce qui concerne les conditions de travail et le principe d'une rémunération décente, elles trouvent à leur côté le Ciane (Collectif interassociatif autour de la naissance).

Dans l'attente de réponses concrètes à ces revendications, une nouvelle journée nationale de grève est programmée en octobre. 